\title{
Quantification of the Specific Translocator Protein Signal of 18F-PBR111 in Healthy Humans: A Genetic Polymorphism Effect on In Vivo Binding
}

\author{
Qi Guo ${ }^{1-3}$, Alessandro Colasanti ${ }^{1,3}$, David R. Owen ${ }^{3}$, Mayca Onega ${ }^{1}$, Aruloly Kamalakaran ${ }^{1}$, Idriss Bennacef ${ }^{4}$, \\ Paul M. Matthews ${ }^{3,4}$, Eugenii A. Rabiner ${ }^{1,2}$, Federico E. Turkheimer ${ }^{2}$, and Roger N. Gunn ${ }^{1,3}$ \\ ${ }^{1}$ Centre for Imaging Sciences, Imanova, London, United Kingdom; ${ }^{2}$ IoP, King's College London, London, United Kingdom; ${ }^{3}$ Division \\ of Brain Sciences, Department of Medicine, Imperial College London, London, United Kingdom; and ${ }^{4}$ GlaxoSmithKline, Brentford, \\ Middlesex, United Kingdom
}

PET is used to image active inflammatory processes by targeting the translocator protein (TSPO). In vitro, second-generation TSPO radioligands, such as PBR111, have been shown to bind to human tissue samples with either high affinity (high-affinity binders, HABs), low affinity (low-affinity binders, LABs), or an intermediate, mixed affinity (mixed-affinity binders, MABs). We previously explained these differences in affinity in human tissue via the rs6971 polymorphism in the TSPO gene and predicted that the specific signal from PET ligands in vivo would vary accordingly. In silico modeling predicted that ${ }^{18} \mathrm{~F}-\mathrm{PBR} 111$ would have a moderate to high specificto-nonspecific ratio in the normal human brain. To test these predictions, we present here the analysis and modeling of ${ }^{18} \mathrm{~F}-\mathrm{PBR} 111$ data in healthy humans. Methods: Twenty-one subjects (9 HABs, 8 MABs, and 4 LABs), 28-62 y old, genotyped for the rs6971 polymorphism, underwent 120-min PET scans with arterial sampling after a bolus injection of ${ }^{18} \mathrm{~F}$-PBR111. Compartmental models and Logan graphical methods enabled estimation of the total volume of distribution $\left(\mathrm{V}_{\mathrm{T}}\right)$ in regions of interest (ROIs). To evaluate the specific signal, we developed 2 methods to estimate the nondisplaceable volume of distribution $\left(\mathrm{V}_{\mathrm{ND}}\right)$ : the first assumed that the in vitro affinity ratio of ${ }^{18} \mathrm{~F}$-PBR111 in HABs relative to LABs (4-fold) is preserved in vivo; the second modeled the difference in the HAB and MAB signals in the context of an occupancy plot. Results: A 2-tissue-compartment model described the data well, and a significant difference was found between the $V_{T}$ of HABs, MABs, and LABs across all ROls examined $(P<0.05)$. We also found a significant correlation between $\mathrm{V}_{\mathrm{T}}$ and age for both HABs and MABs in most ROls. The average $V_{N D}$ estimated by the 2 methods was $1.18 \pm 0.35$ (method $\mathrm{I}: \mathrm{V}_{\mathrm{ND}}=0.93$, method II: $\mathrm{V}_{\mathrm{ND}}=1.42$ ), implying that the ${ }^{18} \mathrm{~F}-\mathrm{PBR} 111$ $\mathrm{BP}_{\mathrm{ND}}$ was $2.78 \pm 0.46$ in $\mathrm{HABs}, 1.48 \pm 0.28$ in MABs, and $0.51 \pm$ 0.17 in LABs and that the in vivo affinity ratio was similar to that measured in vitro. Conclusion: ${ }^{18} \mathrm{~F}-\mathrm{PBR} 111$ shows a high specific signal in the healthy human brain in vivo. A large component of the variability in the signal across subjects is explained by genetic variation and age, indicating that ${ }^{18} \mathrm{~F}-\mathrm{PBR} 111$ can be used for the quantitative assessment of TSPO expression.

Received Feb. 1, 2013; revision accepted May 24, 2013.

For correspondence or reprints contact: Qi Guo, Imanova, 2nd Floor, Burlington Danes Building, Imperial College London, Du Cane Rd., London, U.K. W12 0NN.

E-mail: qi.guo@kcl.ac.uk

Published online Sep. 26, 2013.

COPYRIGHT (C) 2013 by the Society of Nuclear Medicine and Molecular Imaging, Inc.
Key Words: ${ }^{18} \mathrm{~F}-\mathrm{PBR} 111$; TSPO; specific binding; neuroinflammation; rs6971 polymorphism

J Nucl Med 2013; 54:1915-1923

DOI: 10.2967/jnumed.113.121020

$\mathbf{T}$ he inflammatory response within the brain and spinal cord is mediated by microglia, the resident immunocompetent cells of the central nervous system. Microglia become activated after mild to severe neuronal damage resulting from traumatic, inflammatory, degenerative, or neoplastic disease (1) and have therefore been used as an index of neuroinflammatory processes in imaging studies. A method to quantify microglial activation would prove of utility as a diagnostic tool or a pharmacodynamic marker in drug development.

PET has the unique ability to quantify microglial density in the human brain noninvasively by targeting the $18-\mathrm{kDa}$ translocator protein (TSPO), formerly known as the peripheral benzodiazepine receptor (PBR). The TSPO is a nuclear encoded mitochondrial protein, present in high density in macrophages and microglia and elevated in a variety of neuroinflammatory brain diseases, such as Alzheimer disease, multiple sclerosis, stroke, and Huntington disease (2-5). PK11195 ([1-(2-chlorophenyl)- $\mathrm{N}$-methyl- $\mathrm{N}$-(1methylpropyl)-3-isoquinoline carboxamide]), a selective antagonist for the TSPO, has been labeled with ${ }^{11} \mathrm{C}$ and used as a PET radioligand for more than $15 \mathrm{y}$ to image neuroinflammation (6). However, the low brain extraction and poor signal-to-noise ratio of ${ }^{11} \mathrm{C}-(R)-\mathrm{PK} 11195$ images have led to the search for improved TSPO PET imaging agents.

In the past $5 \mathrm{y}$, a substantial number $(>50)$ of second-generation TSPO ligands have been proposed, including ${ }^{18} \mathrm{~F}-\mathrm{PBR} 111,{ }^{11} \mathrm{C}-\mathrm{PBR} 28$, ${ }^{18} \mathrm{~F}-\mathrm{FEPPA} \quad\left({ }^{18} \mathrm{~F}-\mathrm{N}\right.$-2-(2-fluoroethoxy)benzyl)- $N$-(4-phenoxypyridin3-yl)acetamide), and ${ }^{11} \mathrm{C}$-DPA713 $\left({ }^{11} \mathrm{C}-N, N\right.$-diethyl-2-[2-(4-methoxyphenyl)-5,7-dimethyl-pyrazolo[1,5-a]pyrimidin-3-yl]-acetamide) (7-10). Although these ligands were claimed to have increased delivery across the blood-brain barrier or a higher signal-to-noise ratio than ${ }^{11} \mathrm{C}-(R)-\mathrm{PK} 11195$, initial human studies have demonstrated considerable variability in their binding across subjects, including the presence of the so-called nonbinders, healthy subjects who demonstrated very little if any binding of ${ }^{11} \mathrm{C}-\mathrm{PBR} 28(8)$. We have previously demonstrated in vitro, in human tissue samples, that all 


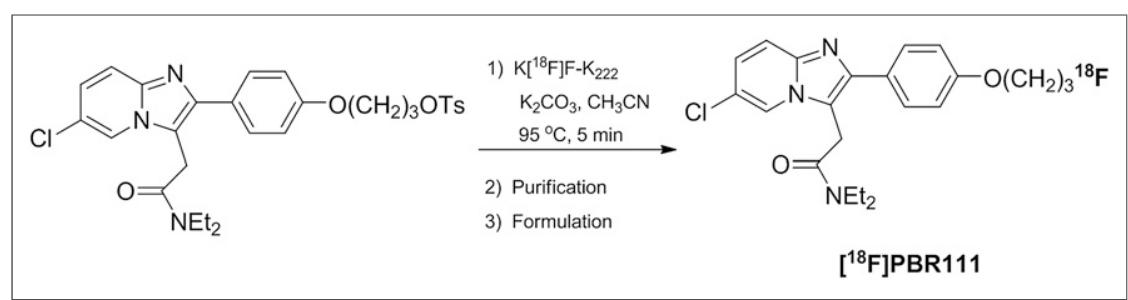

FIGURE 1. Radiosynthesis of ${ }^{18} \mathrm{~F}-\mathrm{PBR} 111$.

second-generation ligands tested bind TSPO with either high affinity (high-affinity binders, HABs), or low affinity (low-affinity binders, LABs), and that some subjects have a mix of high- and low-affinity binding sites (mixed-affinity binders, MABs) $(11,12)$. This finding has retrospectively explained the "nonbinders" phenomenon, as the affinity of ${ }^{11} \mathrm{C}-\mathrm{PBR} 28$ to LABs was only $188 \mathrm{nM}$ (11) and thus no specific binding in these subjects would be expected. The presence of MABs in the population also explained the variability in the PET signal across healthy volunteers, as these subjects may be expected to have significantly lower-affinity binding of the second-generation ligands, from $\mathrm{HAB}$. We went on to demonstrate that these different binding patterns in humans are fully explained by the rs6971 polymorphism in the TSPO gene (13). The frequencies of this polymorphism vary across ethnic groups, with Caucasians having the highest frequency for LABs (HAB:MAB:LAB = 49:42:9) compared with African Americans (56:38:6) and Han Chinese and Japanese (94:6:0.001). Our hypothesis that the rs6971 polymorphism explained the large betweensubject variability seen with second-generation TSPO PET ligands was consistent with early imaging data from our group (14) and with data showing differences between $\mathrm{HAB}$ and $\mathrm{MAB}$ binding for TSPO ligands, ${ }^{18} \mathrm{~F}$-FEPPA, and ${ }^{11} \mathrm{C}$-PBR28 $(9,15)$.

We previously evaluated ${ }^{18} \mathrm{~F}-\mathrm{PBR} 111$ in vivo in the nonhuman primate brain, which demonstrated high delivery, fast kinetics, and high signal-to-noise ratio $\left(\mathrm{BP}_{\mathrm{ND}} \sim 4\right)$, based on the blockade of the ${ }^{18}$ F-PBR111 binding with unlabeled PK11195 (7 mg/kg intravenously) (16). Advances in our in silico biomathematic model

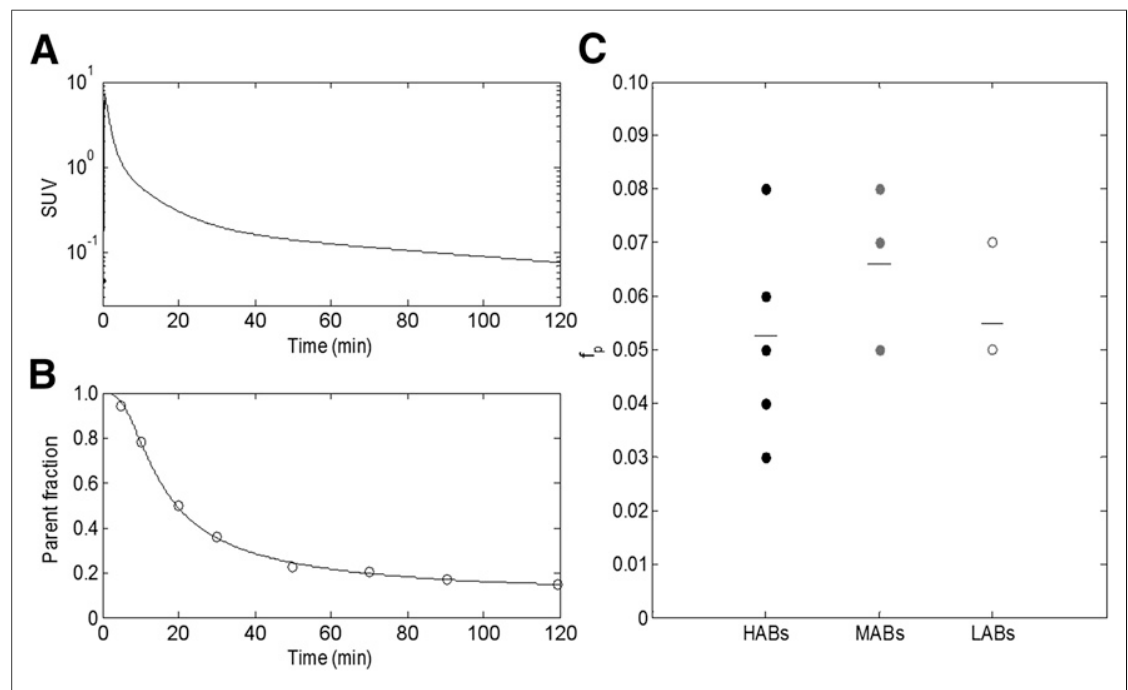

FIGURE 2. Blood measurements. (A) Parent plasma concentration of HAB, fitted with triexponential model after peak. (B) Parent fraction of HAB, fitted with sigmoid parent fraction model. (C) $f_{p}$ for different genetic groups: HABs, MABs, and LABs, with mean estimate for each group. No significant difference was found across groups. predicting the in vivo performance of radioligands in humans (17) enabled us to account for the influence of genetic polymorphisms in the target protein. Our model predicted that ${ }^{18} \mathrm{~F}-\mathrm{PBR} 111$ would show moderate to high specific binding in the healthy human brain across genetic groups (18). Evidence of the ubiquitous expression of TSPO throughout the brain (19) and the global reduction in ${ }^{18} \mathrm{~F}-\mathrm{PBR} 111$ binding after the administration of unlabeled PK11195 in the nonhuman primate (16) indicated that any reference region approach will be confounded by a contribution from specific binding.

Here, the quantification of ${ }^{18} \mathrm{~F}-\mathrm{PBR} 111$ from an initial study in healthy human volunteers is presented, including full kinetic modeling and analysis. We developed mathematic methods that enable estimation of the nondisplaceable volume of distribution $\left(\mathrm{V}_{\mathrm{ND}}\right)$ of ${ }^{18} \mathrm{~F}-\mathrm{PBR} 111$, which then allows calculation of the binding potential (ratio of specific volume of distribution to $\mathrm{V}_{\mathrm{ND}}$ ) in the population (20). We tested the hypothesis that in vivo, the ${ }^{18} \mathrm{~F}-\mathrm{PBR} 111$ signal is dependent on the rs6971 polymorphism in the TSPO gene, and we evaluated the effects of age on binding in the human brain.

\section{MATERIALS AND METHODS}

\section{Human Subjects}

Data were acquired as part of 2 PET studies conducted at Imanova Centre for Imaging Sciences, London. In total, 21 healthy volunteers, aged between 28 and $62 \mathrm{y}$ (mean $\pm \mathrm{SD}, 51 \pm 10 \mathrm{y} ; 14$ women and $7 \mathrm{men}$ ) and having an average body weight of $77.2 \pm 22.4 \mathrm{~kg}$, were included. The studies were approved by the Essex 1 Research Ethics Committee and the Administration of Radioactive Substances Advisory Committee. All subjects gave written informed consent, and their eligibility was confirmed via full medical history, physical and neurologic examinations, routine blood tests, urine analysis, and electrocardiography. The presence of any medical or neurologic illness was an exclusion criterion.

All subjects were genotyped for the rs6971 polymorphism (9 HABs, 8 MABs, and 4 LABs) and underwent a high-resolution T1 MR imaging scan in a Tim Trio 3T scanner and an ${ }^{18} \mathrm{~F}-\mathrm{PBR} 111 \mathrm{PET}$ scan in a Biograph 6 PET/CT scanner (Siemens Healthcare) with Truepoint gantry.

All structural MR images were inspected by an experienced clinical neuroradiologist for unexpected findings of clinical significance or features that might confound PET coregistration or quantitative analysis. In 4 cases ( $2 \mathrm{HABs}$ and $2 \mathrm{MABs}$ ), focal, nonspecific alterations in the signal intensity in the white matter were noted and subjects were referred for further investigations. None of these subjects had past or present neurologic symptoms, and their physical and neurologic examination was unremarkable. Data analyses were repeated after exclusion of these subjects.

\section{Radiopharmaceutical Preparation}

${ }^{18} \mathrm{~F}-\mathrm{PBR} 111$ was labeled with ${ }^{18} \mathrm{~F}$ by a 1-step tosyloxy-for-fluorine nucleophilic aliphatic substitution, followed by purification by semipreparative high-performance liquid chromatography (HPLC) and reformulation (Fig. 1). 


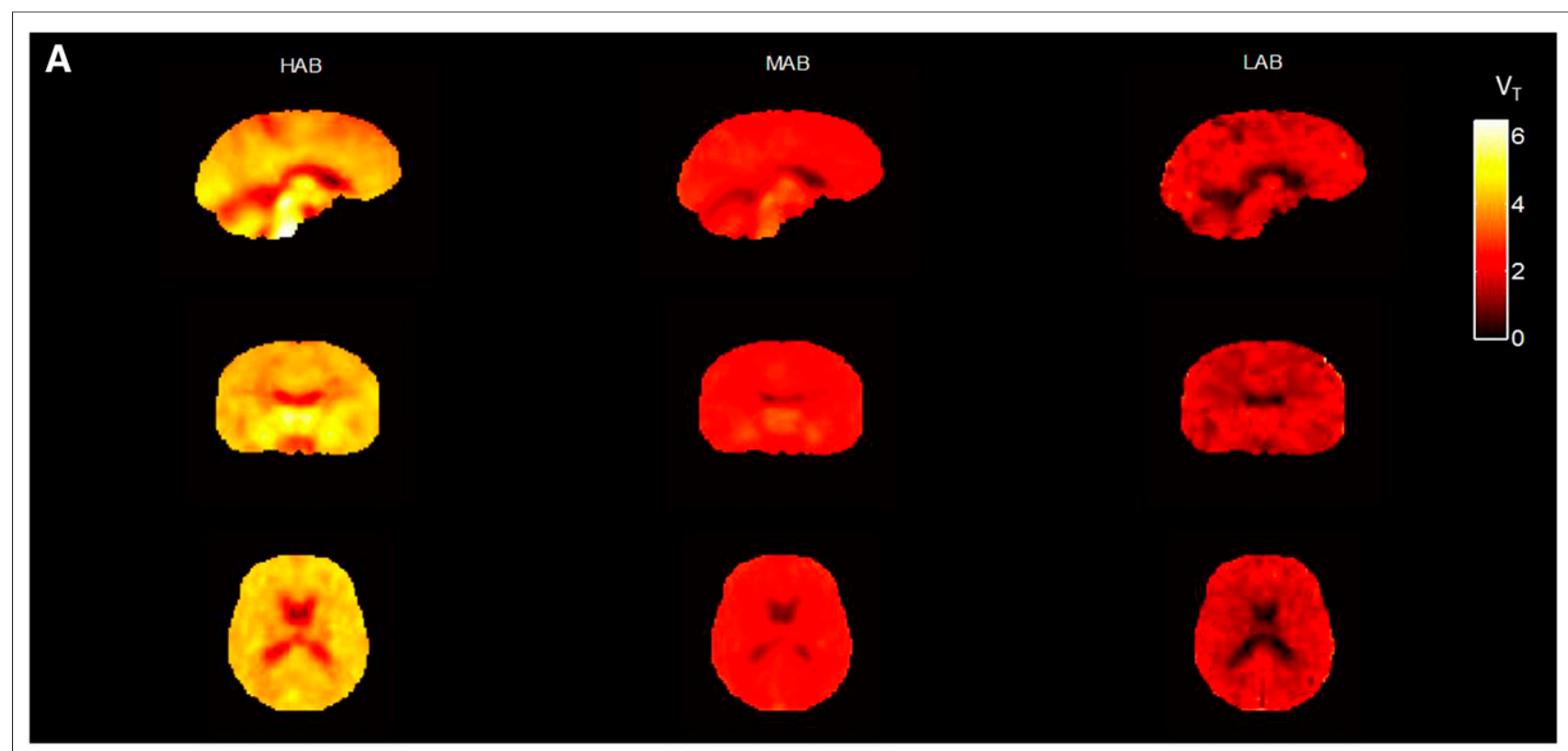

B
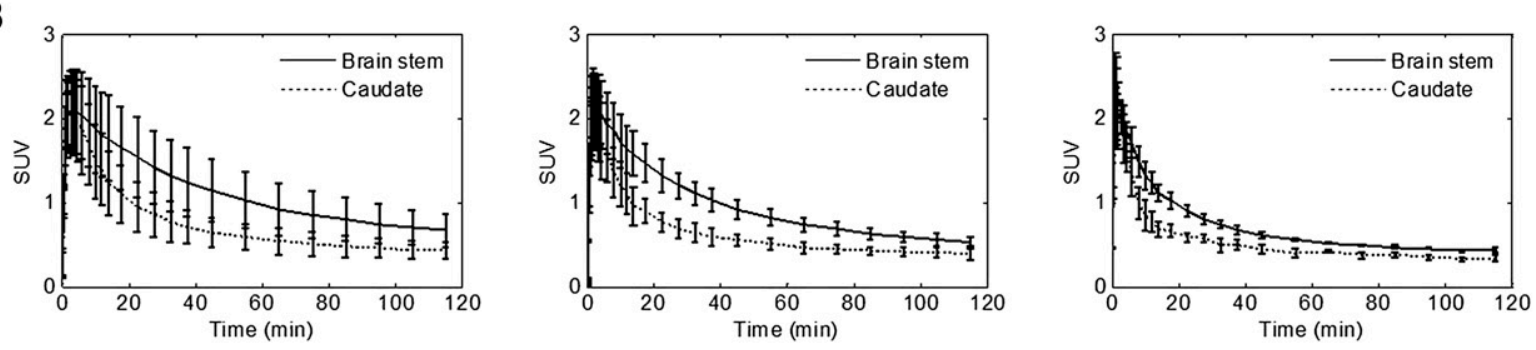

FIGURE 3. $V_{T}$ parametric maps and averaged time-activity curves across genetic groups. (A) Spatially normalized average $V_{T}$ parametric maps for each group. (B) Average time-activity curves in brain stem and caudate for each group. Error bar is SD at each frame.

Synthesis of ${ }^{18}$ F-PBR111 was previously described (21). A fully automated procedure was developed in-house using an Explora GN module (Siemens Healthcare) coupled with a semipreparative HPLC system. No-carrier-added ${ }^{18} \mathrm{~F}$-fluoride was concentrated on a QMA cartridge (Graver Technologies) and eluted with $\mathrm{K}_{2} \mathrm{CO}_{3} / \mathrm{K}_{222}$ in $\mathrm{H}_{2} \mathrm{O} / \mathrm{CH}_{3} \mathrm{CN}$ into the reaction vessel. It was consequently dried by performing 2 successive azeotropic evaporations. The tosyloxy precursor $\left(5-10 \mathrm{mg}\right.$ in $1 \mathrm{~mL}$ of $\mathrm{CH}_{3} \mathrm{CN}$ ) was added to the dry residue, and fluorination was performed at $95^{\circ} \mathrm{C}$ for $5 \mathrm{~min}$. The crude reaction mixture was then diluted with water $(3 \mathrm{~mL})$ and loaded onto the HPLC system for purification on an Eclipse XDB C18 column $(5 \mu \mathrm{m}, 250 \times 9.4 \mathrm{~mm}$; Agilent) with $50 \mathrm{mM}$ ammonium formate buffer $(\mathrm{pH} 4) / \mathrm{CH}_{3} \mathrm{CN}(55: 45, \mathrm{v} / \mathrm{v})$ at $9.5 \mathrm{~mL} / \mathrm{min}$. The fraction containing ${ }^{18} \mathrm{~F}-\mathrm{PBR} 111$ was collected in water $(20 \mathrm{~mL})$ and loaded onto an activated Sep-Pak Classic C18 cartridge (Waters) for reformulation in $10 \%$ ethanol/saline. Finally, a sterile filtration $(0.2-\mu \mathrm{m}$ sterile filter) was performed to deliver the final dose as a sterile and pyrogen-free solution.

Typically, the total ${ }^{18} \mathrm{~F}-\mathrm{PBR} 111$ synthesis procedure, including HPLC purification and Sep-Pak-based formulation, is accomplished in less than $60 \mathrm{~min} .{ }^{18} \mathrm{~F}-\mathrm{PBR} 111,3.57 \pm 0.89 \mathrm{GBq}(>97 \%$ radiochemically pure, $n=21$ ), was obtained starting from $10 \mathrm{GBq}$ of ${ }^{18} \mathrm{~F}$ fluoride with a specific radioactivity of $258.99 \pm 157.38 \mathrm{GBq} / \mu \mathrm{mol}$.

\section{PET Imaging}

${ }^{18} \mathrm{~F}-\mathrm{PBR} 111$ was injected as an intravenous bolus over approximately $20 \mathrm{~s}$ at the start of a 120-min 3-dimensional-mode dynamic
PET acquisition. Injected activities ranged from 142.9 to $182.0 \mathrm{MBq}$ $(165.7 \pm 8.5 \mathrm{MBq}, n=21)$, and the injected mass ranged from 0.14 to $8.66 \mu \mathrm{g}(1.22 \pm 2.18 \mu \mathrm{g})$. PET data were reconstructed using filtered backprojection with corrections for attenuation and scatter (based on a low-dose CT acquisition). Dynamic data were binned into 29 frames (durations: $8 \times 15 \mathrm{~s}, 3 \times 1 \mathrm{~min}, 5 \times 2 \mathrm{~min}, 5 \times 5 \mathrm{~min}$, $8 \times 10 \mathrm{~min}$ ). Arterial blood data were sampled via the radial artery to enable generation of an arterial plasma input function. A continuous sampling system (ABSS Allogg) was used to measure whole-blood activity each second for the first 15 min of each scan. Discrete blood samples were manually withdrawn at $5,10,15,20,25,30,40,50$, $60,70,80,90,100,110$, and $120 \mathrm{~min}$ after scan start to facilitate measurement of whole-blood and plasma activity. Samples taken at $5,10,15,20,30,50,70,90$, and 120 min were also analyzed using HPLC to determine the fraction of parent radioactivity in arterial plasma. The first 3 discrete blood samples were used to calibrate the continuous blood data, and then the continuous and discrete datasets were used to form a whole-blood activity curve covering the duration of the scan. Discrete plasma samples were divided by the corresponding whole-blood samples to form plasma-over-blood data. A constant plasma-over-blood model was fitted. This plasma-overblood value was then multiplied by the whole-blood curve to generate a total plasma curve. Parent fraction data were fitted to a sigmoid model, $f=\left(\left(1-\frac{t^{3}}{t^{3}+10^{a}}\right)^{b}+c\right) /(1+c)$, where $t$ is time and $a, b$, and $c$ are fitted parameters. The resulting fitted parent fraction profile was multiplied by the total plasma curve and then smoothed after the 


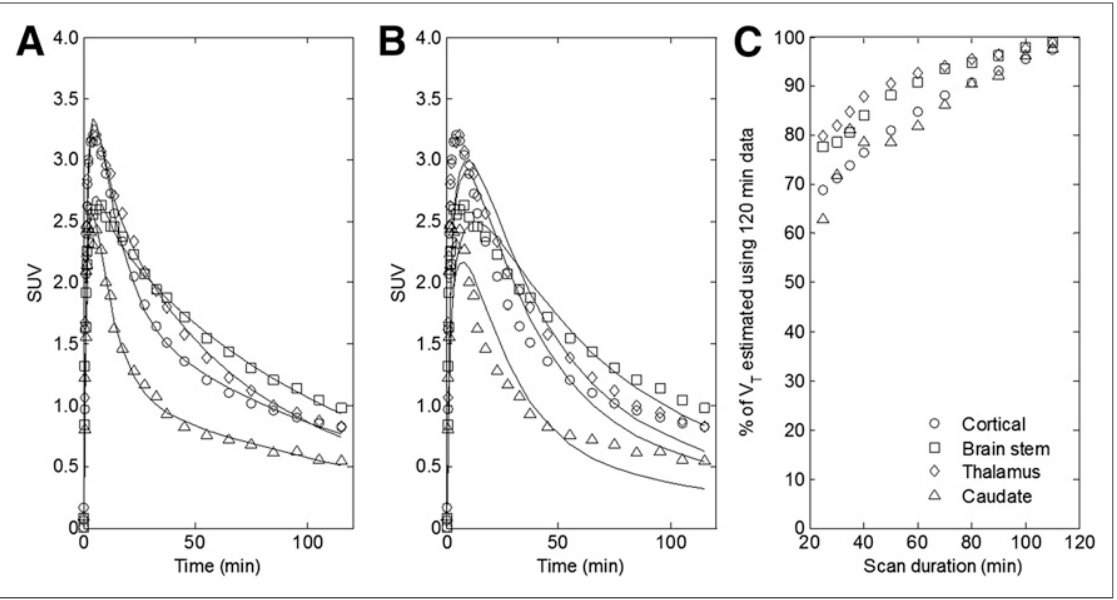

FIGURE 4. Kinetic model fits to HAB time-activity curves and time-stability analysis. (A) Unconstrained 2TC model fit to time-activity curves. (B) Unconstrained 1TC model fit to timeactivity curves. (C) $\mathrm{V}_{\mathrm{T}}$ time-stability assessment for unconstrained $2 \mathrm{TC}$ model. binding effects across subjects (15). $\mathrm{V}_{\mathrm{T}}$ and $\mathrm{V}_{\mathrm{T}} / \mathrm{f}_{\mathrm{p}}$ were compared with the averaged standardized uptake value from 90 to $120 \mathrm{~min}\left(\mathrm{SUV}_{90 \_120 \mathrm{~min}}\right)$. Model fitting and parameter estimation were performed using software implemented in Matlab R2008b (The MathWorks, Inc.).

To investigate the scanning duration required for reproducible estimates of $\mathrm{V}_{\mathrm{T}}$, time stability analysis was performed by analyzing scan durations ranging from 30 to $120 \mathrm{~min}$ in 10-min increments.

\section{Quantification of Specific Binding Signal}

$V_{T}$ is the sum of the specific volume of distribution and $\mathrm{V}_{\mathrm{ND}}$, which is a sum of free and nonspecifically bound tracer. In the absence of a suitable reference region, devoid of TSPO, it is not straightforward to estimate $\mathrm{V}_{\mathrm{ND}}$ and calculate the nondisplaceable bindpeak using a triexponential fit to derive the required parent plasma input function. For each scan, a time delay was fitted and applied to the input function to account for any temporal delay between blood sample measurement and the tomographic measurements of the tissue data. Free fraction in plasma $\left(f_{P}\right)$ was measured through ultrafiltration (Amicon Ultra regenerated cellulose; molecular weight cutoff, $30 \mathrm{kDa}$ [Millex]) in triplicate using Tris buffer $(0.1 \mathrm{M}, \mathrm{pH} 7.4)$ to determine and enable correction for nonspecific binding. Filters were pretreated with $5 \%$ polysorbate 80 to reduce nonspecific binding to the filter membrane (22).

\section{Image Analysis}

Dynamic PET data were corrected for motion via frame-to-frame image registration and aligned with the individual's structural T1weighted MR image using SPM5 (Wellcome Trust Center for Neuroimaging, http://www.fil.ion.ucl.ac.uk/spm) with a mutual-information cost function.

The CIC neuroanatomic atlas (23) was nonlinearly deformed into the individual's space, via mapping of T1-weighted MR imaging data, to obtain a personalized anatomic parcellation of regions of interest (ROIs). Attention focused on regions of moderate- and high-affinity binding based on previous literature (24) - brain stem, thalamus, hippocampus, global cortical regions, and cerebellum-and on representative low-affinity binding regions such as caudate. Because of defluorination of the metabolite from ${ }^{18} \mathrm{~F}-\mathrm{PBR} 111$ (25), ROIs for the frontal, parietal, occipital, and temporal cortex and the cerebellum were eroded to avoid spill-in from uptake outside the brain (26). Each ROI was then applied to the dynamic PET data to derive regional time-activity curves.

\section{Kinetic Analysis}

One- and 2-tissue-compartment (1TC and 2TC, respectively) models using the metabolite-corrected plasma input function were applied to the dynamic PET data using a fixed blood volume correction of $5 \%$. For each ROI examined, the total volume of distribution $\left(\mathrm{V}_{\mathrm{T}}\right)$ was estimated from the rate constants as described previously (27). The Logan graphical method (28), using a plasma input, 5\% fixed blood volume, and linear start time at $35 \mathrm{~min}$, was also applied to estimate the $\mathrm{V}_{\mathrm{T}}$ of each ROI and was further applied at the voxel level to produce a parametric $V_{T}$ map for each subject. In addition to $V_{T}$, $\mathrm{V}_{\mathrm{T}} / \mathrm{f}_{\mathrm{p}}$ was obtained in order to consider correction for plasma protein ing potential $\left(\mathrm{BP}_{\mathrm{ND}}\right)$. We developed 2 methods to estimate $\mathrm{V}_{\mathrm{ND}}$ : method I assumes that the in vitro affinity ratio between HABs and LABs is preserved in vivo, and method II models the difference in the $\mathrm{HAB}$ and $\mathrm{MAB}$ signals in the context of an occupancy plot (29).

Method I. Assuming that $\mathrm{V}_{\mathrm{ND}}$ is the same across subjects and that MABs express equal amounts of the high- and low-affinity binding sites (11), it follows that

$$
\begin{aligned}
& V_{T}^{H}=V_{N D}\left(1+B P_{N D}^{H}\right) \\
& V_{T}^{M}=V_{N D}\left(1+\frac{B P_{N D}^{H}}{2}+\frac{B P_{N D}^{L}}{2}\right) \\
& V_{T}^{L}=V_{N D}\left(1+B P_{N D}^{L}\right)
\end{aligned}
$$

where $V_{T}^{H}, V_{T}^{M}, V_{T}^{L}, B P_{N D}^{H}$, and $B P_{N D}^{L}$ are the total volumes of distribution and binding potentials across ROIs for HABs, MABs, and LABs, respectively. If the affinity ratio between high- and low-affinity binding sites $R^{H / L}$ is known and assuming the densities of the highand low-affinity binding sites are equal,

$$
B P_{N D}^{H}=R^{H / L} B P_{N D}^{L} .
$$

$$
\begin{array}{rll}
V_{T}^{H} & =V_{N D}\left(1+B P_{N D}^{H}\right) \\
V_{T}^{M} & =V_{N D}\left(1+\frac{B P_{N D}^{H}}{2}\left[1+\frac{1}{R^{H / L}}\right]\right), & \text { Eq. } 3 \\
V_{T}^{L} & =V_{N D}\left(1+\frac{B P_{N D}^{H}}{R^{H / L}}\right) .
\end{array}
$$

The in vitro affinity ratio between high- and low-affinity binding sites for ${ }^{18} \mathrm{~F}-\mathrm{PBR} 111$ has been previously estimated in vitro as $R^{H / L}=4.0$ (12). Thus, $\mathrm{V}_{\mathrm{ND}}$ and $B P_{N D}^{H}$ for each ROI can be estimated by directly fitting the $V_{T}^{H}, V_{T}^{M}$, and $V_{T}^{L}$ data obtained across different ROIs to Equation 3.

Method II. Inspired by prior work estimating drug occupancy using graphical methods $(29,30)$, we can also estimate the $\mathrm{V}_{\mathrm{ND}}$ by modeling the difference in the $\mathrm{HAB}$ and $\mathrm{MAB}$ signals without needing to make any
Substituting Equation 2 into Equation 1 yields 
assumptions about the affinity ratio between high- and low-affinity binding sites. This allows derivation of the following equation,

$$
V_{T}^{H}-V_{T}^{M}=\Delta \cdot\left(V_{T}^{H}-V_{N D}\right)
$$

where $\Delta=\frac{1}{2}\left(1-\frac{B_{\max }^{L}}{B_{\max }^{H}} \frac{K_{D}^{H}}{K_{D}^{L}}\right)$ under the assumption that MABs express $50 \%$ of each of the high- and low-affinity binding sites. $B_{\max }^{H}$, $B_{\text {max }}^{L}, K_{D}^{H}$, and $K_{D}^{L}$ are the TSPO density and equilibrium dissociation constant for HABs and LABs, respectively. Thus, by plotting $V_{T}^{H}-V_{T}^{M}$ against $V_{T}^{H}$ and performing a linear regression, $V_{N D}$ can be estimated as the $x$-intercept.

\section{Statistical Analysis}

The Akaike information criterion (AIC) was used to evaluate the goodness of fit of the compartmental models (31). The identifiability (\%) of the rate constants, expressed as the SE of their estimated values, was calculated from the diagonal of the covariance matrix (32). Identifiability (\%) of $\mathrm{V}_{\mathrm{T}}$ was calculated from the covariance matrix using the generalized form of the error propagation equation (33), where correlations among rate constants were considered. The Pearson correlation coefficient was used to estimate the relationship between $\mathrm{V}_{\mathrm{T}}$ and age within each genetic group. Both 2-sample $t$ testing and analysis of covariance were used to evaluate the difference between the genetic groups, with analysis of covariance correcting for age.

\section{RESULTS}

\section{Plasma Analysis}

In plasma, there was a peak in the concentration of ${ }^{18} \mathrm{~F}-\mathrm{PBR} 111$ at about $1 \mathrm{~min}$ followed by a rapid decrease, which was well described by a triexponential model (Fig. 2A). One metabolite appeared quickly after parent administration. The parent compound accounted for about $40 \%$ of the total concentration in plasma at $30 \mathrm{~min}$, and the parent fraction further reduced and reached between $9.3 \%$ and $36.4 \%(20.1 \% \pm 8.7 \%)$ at $120 \mathrm{~min}$ (Fig. 2B). The metabolite has been previously identified as a $3-{ }^{18}$ F-fluoropropionic acid, which is more polar than the parent compound. Bone uptake was observed with ${ }^{18} \mathrm{~F}-\mathrm{PBR} 111$, consistent with defluorination reported for this metabolite in the literature (25). The metabolite profile did not vary across genetic groups. $f_{p}$ was measured for 8 HABs $(0.05 \pm 0.02), 6$ MABs $(0.07 \pm 0.02)$, and 4 LABs $(0.06 \pm 0.01)$. No significant difference was found in $f_{p}$ across the different genetic groups (Fig. 2C).

\section{Kinetic Analysis}

After injection, the tracer readily entered the brain and showed widespread distribution, with slightly higher signal in the thalamus, brain stem, and hippocampus, followed by other cortical regions and the cerebellum, and was lowest in the striatum, consistent with previous findings with ${ }^{11} \mathrm{C}-\mathrm{PBR} 28$ (24). The concentration of the ligand in tissue peaked at around 2 min for all subjects and washed out with different elimination rates corresponding to the different genetic groups (Fig. 3). In HABs, the concentration in a high-affinity binding region such as brain stem decreased to $50 \%$ of the peak at $60 \mathrm{~min}$, whereas in MABs and LABs the washout was faster and the concentration decreased to $50 \%$ of the peak at 40 and $20 \mathrm{~min}$, respectively.

2TC and 1TC fits to the time-activity curves across ROIs in a representative $\mathrm{HAB}$ subject are shown in Figures $4 \mathrm{~A}$ and $4 \mathrm{~B}$, respectively. The $2 \mathrm{TC}$ described the kinetics well and provided a better fit than the $1 \mathrm{TC}$ in all regions $\left(\mathrm{AIC}_{1 \mathrm{TC}}=-4.08 \pm 20.90\right.$ 


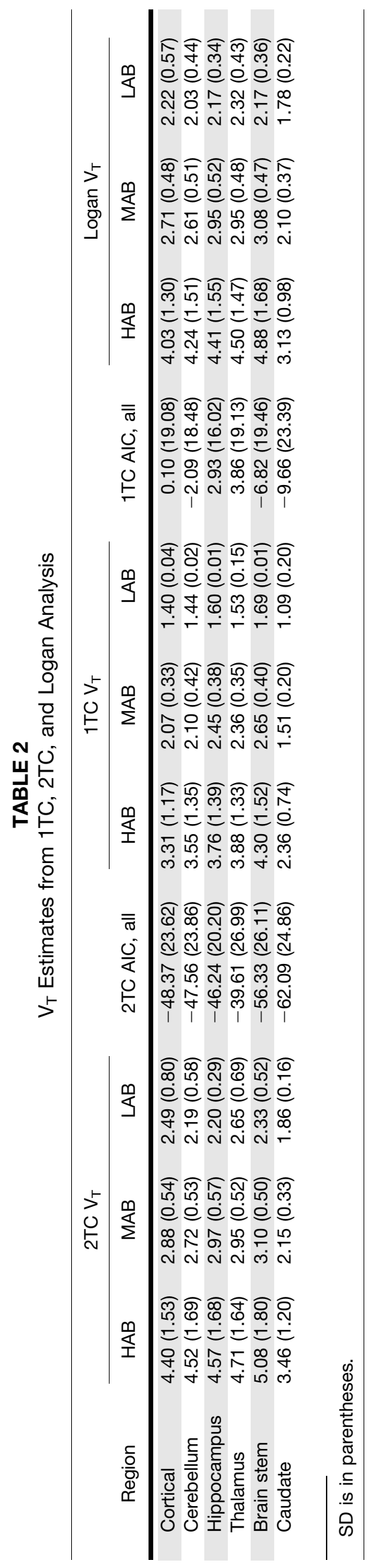

and $\mathrm{AIC}_{2 \mathrm{TC}}=-51.13 \pm 25.52$ ). Rate constants estimated from the 2TC model are listed in Table 1. The 2TC demonstrated good identifiability of the rate constants and $\mathrm{V}_{\mathrm{T}}$ (covariance $<5 \%$ ). The Logan graphical method was also used to estimate the $\mathrm{V}_{\mathrm{T}}$ in each $\mathrm{ROI}$ and at the voxel level to produce parametric maps. The ROIbased $\mathrm{V}_{\mathrm{T}}$ from 1TC, 2TC, and Logan for each genetic group are listed for comparison in Table 2. $\mathrm{V}_{\mathrm{T}}$ estimates from the $2 \mathrm{TC}$ and Logan were in good agreement with $\mathrm{V}_{\mathrm{T} \_ \text {logan }}=0.91 \mathrm{~V}_{\mathrm{T} \_2 \mathrm{TC}}+0.19$ $\left(r^{2}=0.98\right)$. Based on better goodness of fit and good identifiability, the 2TC was selected as the model to use for further ROI analysis.

The time stability analysis of the 2TC model demonstrated an increase in $\mathrm{V}_{\mathrm{T}}$ extending beyond the 120-min scan duration used in our studies (Fig. 4C). This effect was present in all brain regions and for all genetic groups. A scan duration of $60 \mathrm{~min}$ would lead to $10 \%-20 \%$ underestimation of $\mathrm{V}_{\mathrm{T}}$ in comparison to a value obtained from the full 120-min acquisition.

\section{Genetic Influence on In Vivo Binding Signal}

Figure 5 compares the $\mathrm{SUV}_{90 \_120 \mathrm{~min}}, \mathrm{~V}_{\mathrm{T}}$, and $\mathrm{V}_{\mathrm{T}} / \mathrm{f}_{\mathrm{p}}$ values in gray matter-masked ROIs across different genetic groups. Although a difference was observed among groups for all 3 parameters, $S_{U V} V_{90} 120$ min did not reach significance whereas significant differences were found for $\mathrm{V}_{\mathrm{T}}$ among all groups (HABs vs. MABs: $P<0.038$; HABs vs. LABs: $P<0.011$; and MABs vs. LABs: $P<0.032)$. Accounting for plasma free fraction $\left(\mathrm{V}_{\mathrm{T}} / \mathrm{f}_{\mathrm{p}}\right)$ did not reduce the variability between subjects. The $V_{T} / f_{p}$ of HABs was significantly different from that of MABs and LABs across all regions, but significant differences between MABs and LABs were found in only a few regions such as thehippocampus and brain stem. Thus, only $\mathrm{V}_{\mathrm{T}}$ is considered in the following analyses. The average absolute difference in $V_{T}$ between HABs and MABs is $1.54 \pm 0.23$, which is similar to the average absolute difference observed between MABs and LABs $(1.14 \pm 0.17)$. This finding is consistent with the hypothesis that MABs express half the high-affinity sites and half the low-affinity sites, in which case $V_{T}^{H}-V_{T}^{M}=V_{T}^{M}-V_{T}^{L}=\left(V_{S}^{H}-V_{S}^{L}\right) / 2$.

An averaged spatially normalized $V_{T}$ parametric map for each genetic group was produced by warping each individual's $V_{T}$ parametric map to the Montreal Neurological Institute space (http://www.bic.mni.mcgill.ca) and then averaging them within each group (Fig. 3). A global difference in $\mathrm{V}_{\mathrm{T}}$ was demonstrated across the different genetic groups consistent with the results from the ROI analyses.

\section{Relationship with Age}

Within each genetic group, there was still substantial variability in $V_{T}$, especially in HABs. We found a significant correlation between age and $\mathrm{V}_{\mathrm{T}}$ in all ROIs for HABs and in most ROIs for MABs. The linear regression slope in HABs (slope $=0.09$ ) was about 2-fold higher than the slope estimated for MABs (0.04) (Supplemental Table 1; supplemental materials are available at http://jnm.snmjournals.org), consistent with the expectation of increased age effects in subjects with higher specific binding (Fig. 6 ). Results in LABs were not included here because of the small sample size $(n=4)$ and limited age range (44-56 y old). When analysis of covariance was used to correct for the age effect within each binding class, the SEM in HABs was reduced by $40 \%$ (Supplemental Table 2).

\section{Specific TSPO Binding Signal}

By fitting the average $V_{T}$ of HABs, MABs, and LABs to Equation 3 all at once across all regions under the assumption that 


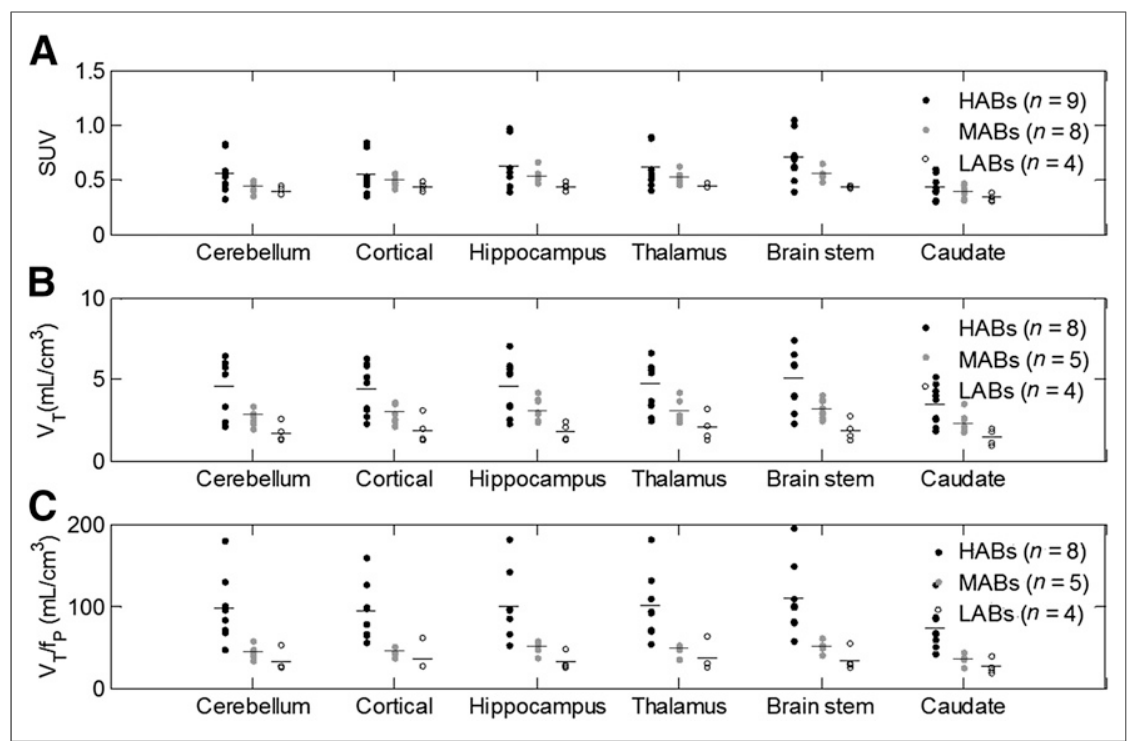

FIGURE 5. Comparison of $\mathrm{SUV}_{90} 120 \mathrm{~min}, \mathrm{~V}_{\mathrm{T}}$, and $\mathrm{V}_{\mathrm{T}} / \mathrm{f}_{\mathrm{p}}$ values across genetic groups. Significant difference was found with $\mathrm{V}_{\mathrm{T}}$ between groups. (HABs vs. MABs: $P<0.038$; HABs vs. LABs: $P<$ 0.011 ; and MABs vs. LABs: $P<0.032$ ).

nonspecific binding was the same in all subjects, we estimated a $\mathrm{V}_{\mathrm{ND}}$ of 0.93 (Fig. 7A). $\mathrm{V}_{\mathrm{ND}}$ was also estimated by the graphical plot (method II, Eq. 4), without making any assumptions about the in vivo affinity ratio between high- and low-affinity binding sites, as the $x$-intercept value of 1.42 (Fig. 7B). Based on the consistency of the 2 methods, we obtained an average estimate of 1.18 for $\mathrm{V}_{\mathrm{ND}}$, which enabled calculation of ${ }^{18} \mathrm{~F}-\mathrm{PBR} 111 \mathrm{BP}_{\mathrm{ND}}$ in the normal human brain: $2.78 \pm 0.46$ in HABs, $1.48 \pm 0.28$ in MABs, and $0.51 \pm 0.17$ in LABs in the ROIs examined. Data analyses were repeated after exclusion of the 4 subjects with nonspecific findings in the white matter, and the results remained unaltered.

\section{DISCUSSION}

This paper has considered quantification of ${ }^{18} \mathrm{~F}-\mathrm{PBR} 111$ in the healthy human brain. We previously identified a strong effect of the rs6971 polymorphism in the TSPO gene on the binding affinity of PBR111 to TSPO in human tissue in vitro (12). Here, we com-

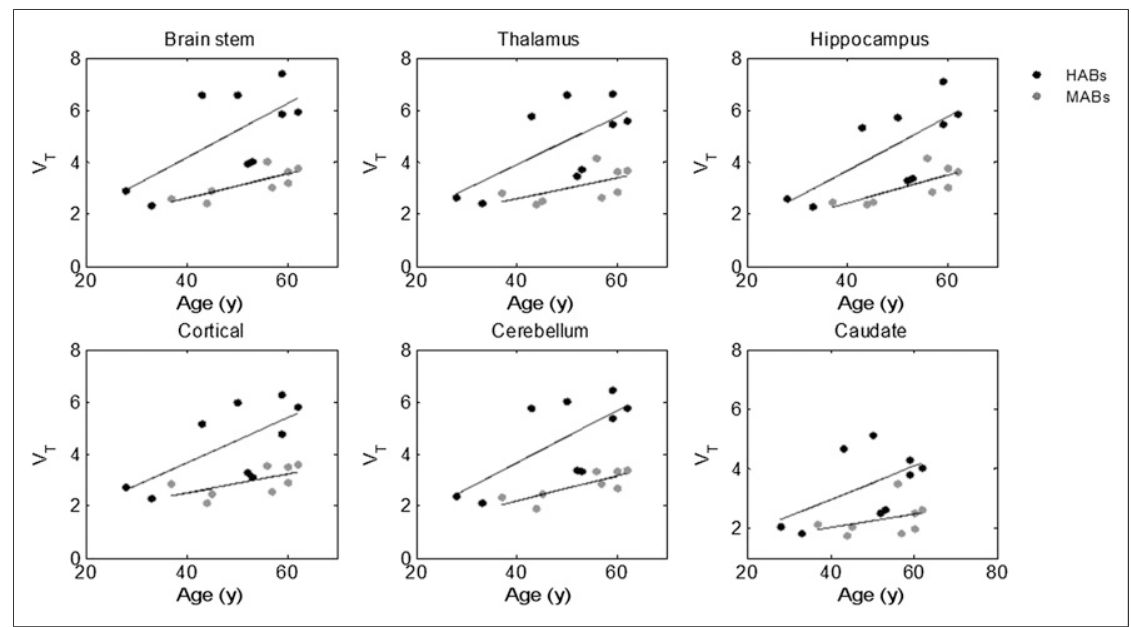

FIGURE 6. Correlation between $\mathrm{V}_{\mathrm{T}}$ and age in HABs and MABs. pared the binding of ${ }^{18} \mathrm{~F}-\mathrm{PBR} 111$ in vivo in these genetic groups, using 3 outcome measures, namely $\mathrm{SUV}_{90 \_120 \mathrm{~min}}, \mathrm{~V}_{\mathrm{T}}$, and $\mathrm{V}_{\mathrm{T}} / \mathrm{f}_{\mathrm{p}}$. Significant differences were observed between HABs, MABs, and LABs with $V_{T}$ and $\mathrm{V}_{\mathrm{T}} / \mathrm{f}_{\mathrm{p}}$, which are predicted by the genetic status of the subjects. In our data, $f_{p}$ did not differ across genetic groups in the healthy subjects and the variability between subjects in $\mathrm{V}_{\mathrm{T}}$ was not reduced after correction for the free fraction in plasma. Therefore, $\mathrm{V}_{\mathrm{T}}$ estimated from an unconstrained 2TC model was chosen as an appropriate measure to quantify the total binding of TSPO, although the relevance of $f_{P}$ correction should be evaluated for individual pathologic groups. Differences in $\mathrm{SUV}_{90-120}$ min were observed between genetic groups similar to the kinetics-based analysis methods, although the differences did not reach significance. This may indicate that static scans in a clinical setting would be viable, but more samples will be required to test this possibility fully.

Within each genetic group, there was still variability in $V_{T}$ across subjects, especially in HABs. We found that this variability can be partly explained by a correlation between age and binding. By using analysis of covariance to correct for the effect of age, the SEM $\mathrm{V}_{\mathrm{T}}$ for HABs was reduced by $40 \%$. Thus, age effect should be considered carefully in TSPO study design either by recruiting subjects within a narrow age range or by applying an appropriate correction for age-related changes. Although the relationship between $\mathrm{V}_{\mathrm{T}}$ and age across the full age range is unlikely to be linear, a linear regression was used as a first-order approximation. We found the slope of HABs to be about 2-fold higher than that seen for MABs, as is consistent with the previously measured affinity ratio between high- and low-specificity binding sites in vitro. We also considered the effect of the variation of injected mass on the $V_{T}$ values. However, no significant correlation was found between these 2 measures.

In silico models based on in vitro and nonhuman primate data predicted a significant specific binding component for ${ }^{18} \mathrm{~F}-\mathrm{PBR} 111$ in the healthy human brain (18). To evaluate the specific binding signal directly, we developed 2 new methods to estimate the $\mathrm{V}_{\mathrm{ND}}$, with the first assuming that the in vitro affinity ratio is preserved in vivo and the second modeling the difference in the HAB and MAB signals in the context of an occupancy plot. Both methods provided a similar estimate for $\mathrm{V}_{\mathrm{ND}}$ (method I: $\mathrm{V}_{\mathrm{ND}}=0.93$, method II: $\mathrm{V}_{\mathrm{ND}}=1.42$ ). An average $\mathrm{V}_{\mathrm{ND}}$ value of around 1.18 implies that the in vivo affinity was similar to that measured in vivo and leads to binding potential estimates ranging from 2.03 to 3.45 in $\mathrm{HABs}, 1.03$ to 1.82 in MABs, and 0.25 to 0.76 in LABs. This moderate to high specific binding signal given by the ligand across the whole brain is consistent with the ubiquitous ex- 


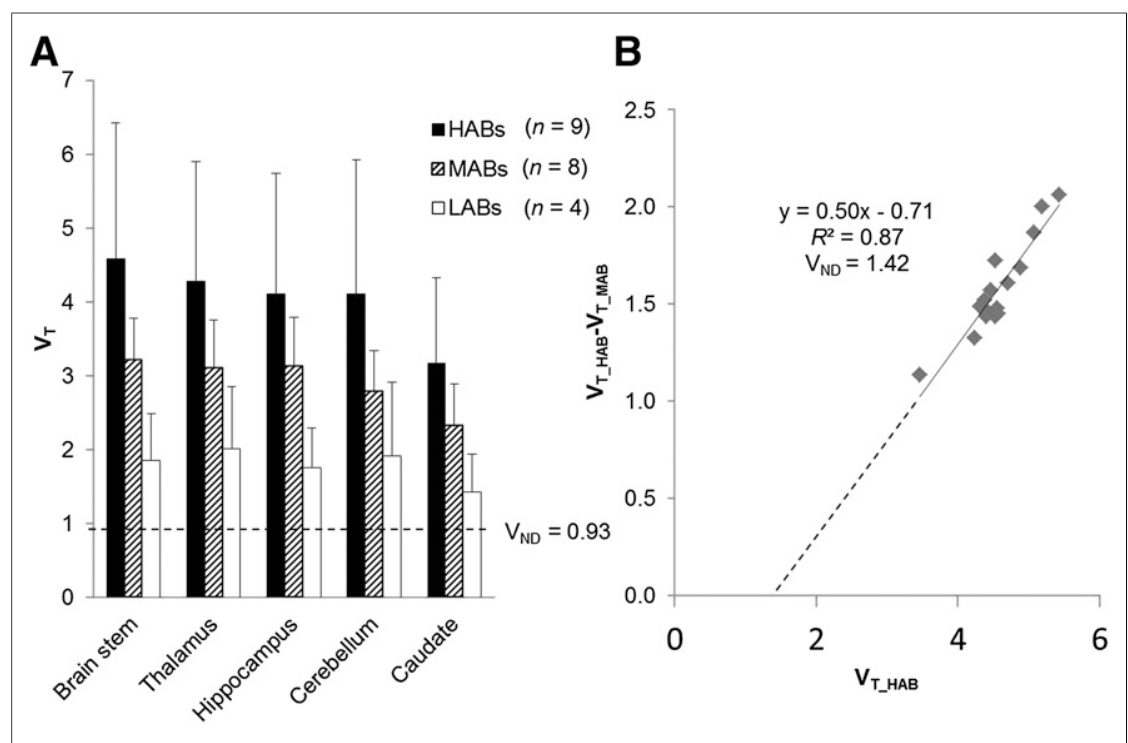

FIGURE 7. Estimation of $\mathrm{V}_{\mathrm{ND}}$ and $\mathrm{BP}_{\mathrm{ND}}$ of ${ }^{18} \mathrm{~F}-\mathrm{PBR} 111$. (A) Method $\mathrm{I}$, assuming in vitro affinity ratio is preserved in vivo. (B) Method II, graphical plot with $x$-intercept representing $V_{N D}$.

\section{CONCLUSION}

${ }^{18}$ F-PBR111 PET showed a measurable and quantifiable specific signal in the healthy human brain for TSPO. The intersubject variability in the PET signal for healthy volunteers can be attributed to the genetic variation at the rs6971 locus and subject age. With these effect accounted for, the ligand can be used for the quantitative assessment of TSPO expression related to neuroinflammatory processes in the brain. The ${ }^{18} \mathrm{~F}$ label provides an advantage for its use in future clinical trials, but quantification currently requires arterial input methods, which limit its use to centers with adequate infrastructure and expertise. In addition, there is an element of defluorination present, and care should be taken when regions close to bone are evaluated (e.g., cortical gray matter).

\section{DISCLOSURE}

pression of TSPO throughout the brain and demonstrates that no obvious reference region exists for ${ }^{18} \mathrm{~F}-\mathrm{PBR} 111$.

The 2TC model fits the time-activity curves well except for a slight underestimation of the tail. This observation agrees with the interpretation of the time stability analysis that shows $V_{T}$ to increase with scan duration. This increase could be due to some small irreversible component, with one possibility being that radiometabolite is getting into the brain. A similar observation has been made with other second-generation TSPO tracers, such as ${ }^{11} \mathrm{C}-\mathrm{PBR} 28,{ }^{11} \mathrm{C}-\mathrm{DPA} 713$, and ${ }^{18} \mathrm{~F}-\mathrm{PBR} 06$ in humans $(10,24,34)$. Because the increase in $\mathrm{V}_{\mathrm{T}}$ of ${ }^{18} \mathrm{~F}-\mathrm{PBR} 111$ was global, as with other tracers, the scan can likely be shortened to $60 \mathrm{~min}$, allowing for a consistent underestimation of less than $20 \%$. However, when different groups are compared, analysis should always be performed with the data from the same scan durations, and the degree of underestimation in different clinical populations needs to be evaluated.

Compared with other TSPO tracers that have been evaluated in humans, such as ${ }^{11} \mathrm{C}-(R)-\mathrm{PK} 11195$ and ${ }^{11} \mathrm{C}-\mathrm{PBR} 28,{ }^{18} \mathrm{~F}-\mathrm{PBR} 111$ showed similar uptake. To demonstrate the specific binding of ${ }^{18} \mathrm{~F}-\mathrm{PBR} 111$, we analyzed both the in silico/in vitro and the in vivo data. Previously, we successfully developed and validated a biomathematic model that predicts the in vivo performance of radiotracers directly from in silico/in vitro data, that is, molecular volume, lipophilicity, affinity, free fractions, and target density (18). Further incorporation of genetic polymorphism status allowed the model to predict that ${ }^{18} \mathrm{~F}-\mathrm{PBR} 111$ should have higher $\mathrm{BP}_{\mathrm{ND}}(\sim 0.74$ and 0.44 in HABs and MABs) than ${ }^{11} \mathrm{C}-(R)$-PK11195 $(\sim 0.2)$ properties (18). In vivo, ${ }^{18} \mathrm{~F}-\mathrm{PBR} 111$ also demonstrated a higher $\mathrm{BP}_{\mathrm{ND}}$ of about $0.51-2.78$ based on the estimated $\mathrm{V}_{\mathrm{ND}}$ of 1.18 as compared with the binding potential of ${ }^{11} \mathrm{C}-(R)-\mathrm{PK} 11195$, which has been estimated previously relative to cortical gray matter as $0.1-0.5$ in healthy brains using a SuperPK method (35). Because ${ }^{11} \mathrm{C}-(R)-$ PK11195 does not bind to TSPO with different affinities and no blocking data are available, it is not clear whether the binding potential estimated for ${ }^{11} \mathrm{C}-(R)-\mathrm{PK} 11195$ is biased by a specific signal in the reference regions used. However, overall, the in vivo findings are consistent with the in silico biomathematic predictions.
The costs of publication of this article were defrayed in part by the payment of page charges. Therefore, and solely to indicate this fact, this article is hereby marked "advertisement" in accordance with 18 USC section 1734. This work was financially supported by grant from Medical Research Council (G0900891). No other potential conflict of interest relevant to this article was reported.

\section{ACKNOWLEDGMENTS}

We acknowledge Yvonne Lewis, Awet Tewolde, Rahul Dimber, Michelle Cunneen, James Anscombe, and Graham Searle for collecting and analyzing data, and Andrea Thiele, Thomas Dyrks, and Ron Weiner for providing previous data on ${ }^{18} \mathrm{~F}-\mathrm{PBR} 111$.

\section{REFERENCES}

1. Banati RB. Visualising microglial activation in vivo. Glia. 2002;40:206-217.

2. Edison P, Archer HA, Gerhard A, et al. Microglia, amyloid, and cognition in Alzheimer's disease: an $\left[{ }^{11} \mathrm{C}\right](\mathrm{R}) \mathrm{PK} 11195-\mathrm{PET}$ and $\left[{ }^{11} \mathrm{C}\right] \mathrm{PIB}-\mathrm{PET}$ study. Neurobiol Dis. 2008;32:412-419.

3. Gerhard A, Schwarz J, Myers R, Wise R, Banati RB. Evolution of microglial activation in patients after ischemic stroke: a $\left[{ }^{11} \mathrm{C}\right](\mathrm{R})-\mathrm{PK} 11195$ PET study. Neuroimage. 2005;24:591-595.

4. Debruyne JC, Versijpt J, Van Laere KJ, et al. PET visualization of microglia in multiple sclerosis patients using $\left[{ }^{11}\right.$ C $]$ PK11195. Eur J Neurol. 2003;10:257264.

5. Pavese N, Gerhard A, Tai YF, et al. Microglial activation correlates with severity in Huntington disease: a clinical and PET study. Neurology. 2006; 66:1638-1643.

6. Cagnin A, Kassiou M, Meikle SR, Banati RB. Positron emission tomography imaging of neuroinflammation. Neurotherapeutics. 2007;4:443-452.

7. Chauveau F, Boutin H, Van Camp N, Dolle F, Tavitian B. Nuclear imaging of neuroinflammation: a comprehensive review of $\left[{ }^{11} \mathrm{C}\right] \mathrm{PK} 11195$ challengers. Eur J Nucl Med Mol Imaging. 2008;35:2304-2319.

8. Kreisl WC, Fujita M, Fujimura Y, et al. Comparison of $\left[{ }^{11} \mathrm{C}\right]-(\mathrm{R})-\mathrm{PK} 11195$ and $\left[{ }^{11} \mathrm{C}\right] \mathrm{PBR} 28$, two radioligands for translocator protein $(18 \mathrm{kDa})$ in human and monkey: implications for positron emission tomographic imaging of this inflammation biomarker. Neuroimage. 2010;49:2924-2932.

9. Mizrahi R, Rusjan PM, Kennedy J, et al. Translocator protein (18 kDa) polymorphism (rs6971) explains in-vivo brain binding affinity of the PET radioligand $\left[{ }^{18}\right.$ F]-FEPPA. J Cereb Blood Flow Metab. 2012;32:968-972.

10. Endres CJ, Pomper MG, James M, et al. Initial evaluation of ${ }^{11} \mathrm{C}-\mathrm{DPA}-713$, a novel TSPO PET ligand, in humans. J Nucl Med. 2009;50:1276-1282. 
11. Owen DR, Howell OW, Tang SP, et al. Two binding sites for $\left[{ }^{3} \mathrm{H}\right] \mathrm{PBR} 28$ in human brain: implications for TSPO PET imaging of neuroinflammation. J Cereb Blood Flow Metab. 2010;30:1608-1618.

12. Owen DR, Gunn RN, Rabiner EA, et al. Mixed-affinity binding in humans with 18-kDa translocator protein ligands. J Nucl Med. 2011;52:24-32.

13. Owen DR, Yeo AJ, Gunn RN, et al. An 18-kDa translocator protein (TSPO) polymorphism explains differences in binding affinity of the PET radioligand PBR28. J Cereb Blood Flow Metab. 2012;32:1-5.

14. Guo Q, Colasanti A, Onega M, et al. Quantification of ${ }^{18}$ F-PBR111 PET for TSPO imaging in humans [abstract]. J Cereb Blood Flow Metab. 2012;32(suppl):S185.

15. Kreisl WC, Jenko KJ, Hines CS, et al. A genetic polymorphism for translocator protein $18 \mathrm{kDa}$ affects both in vitro and in vivo radioligand binding in human brain to this putative biomarker of neuroinflammation. J Cereb Blood Flow Metab. 2013;33:53-58.

16. Carson R, Weinzimmer D, Koren A, et al. Comparison of 3 F-18 TSPO ligands in non-human primates [abstract]. $J$ Nucl Med. 2010;51(suppl):153P.

17. Guo Q, Brady M, Gunn RN. A biomathematical modeling approach to central nervous system radioligand discovery and development. J Nucl Med. 2009;50:1715-1723.

18. Guo Q, Owen DR, Rabiner EA, Turkheimer FE, Gunn RN. Identifying improved TSPO PET imaging probes through biomathematics: the impact of multiple TSPO binding sites in vivo. Neuroimage. 2012;60:902-910.

19. Chen MK, Baidoo K, Verina T, Guilarte TR. Peripheral benzodiazepine receptor imaging in CNS demyelination: functional implications of anatomical and cellular localization. Brain. 2004;127:1379-1392.

20. Innis RB, Cunningham VJ, Delforge J, et al. Consensus nomenclature for in vivo imaging of reversibly binding radioligands. J Cereb Blood Flow Metab. 2007; 27:1533-1539.

21. Dollé F, Hinnen F, Damont A, et al. Radiosynthesis of $\left[{ }^{18} \mathrm{~F}\right] \mathrm{PBR} 111$, a selective radioligand for imaging the translocator protein $(18 \mathrm{kDa})$ with PET. $J$ Labelled Comp Radiopharm. 2008;51:435-439.

22. Lee KJ, Mower R, Hollenbeck T, et al. Modulation of nonspecific binding in ultrafiltration protein binding studies. Pharm Res. 2003;20:1015-1021.

23. Tziortzi AC, Searle GE, Tzimopoulou $S$, et al. Imaging dopamine receptors in humans with $\left[{ }^{11} \mathrm{C}\right]-(+)-\mathrm{PHNO}$ : dissection of D3 signal and anatomy. Neuroimage. 2011;54:264-277.
24. Fujita M, Imaizumi M, Zoghbi SS, et al. Kinetic analysis in healthy humans of a novel positron emission tomography radioligand to image the peripheral benzodiazepine receptor, a potential biomarker for inflammation. Neuroimage. 2008;40:43-52.

25. Fookes CJ, Pham TQ, Mattner F, et al. Synthesis and biological evaluation of substituted $\left[{ }^{18} \mathrm{~F}\right]$ imidazo[1,2-a]pyridines and $\left[{ }^{18} \mathrm{~F}\right]$ pyrazolo $[1,5$-a $]$ pyrimidines for the study of the peripheral benzodiazepine receptor using positron emission tomography. J Med Chem. 2008;51:3700-3712.

26. Carson RE, Wu Y, Lang L, et al. Brain uptake of the acid metabolites of F-18labeled WAY 100635 analogs. J Cereb Blood Flow Metab. 2003;23:249-260.

27. Gunn RN, Lammertsma AA, Grasby PM. Quantitative analysis of [carbonyl- ${ }^{11} \mathrm{C}$ ] WAY-100635 PET studies. Nucl Med Biol. 2000;27:477-482.

28. Logan J, Fowler JS, Volkow ND, et al. Graphical analysis of reversible radioligand binding from time-activity measurements applied to $\left[\mathrm{N}-{ }^{11} \mathrm{C}-m e t h y l\right]-$ (-)-cocaine PET studies in human subjects. J Cereb Blood Flow Metab. 1990;10:740-747.

29. Cunningham VJ, Rabiner EA, Slifstein M, Laruelle M, Gunn RN. Measuring drug occupancy in the absence of a reference region: the Lassen plot re-visited. J Cereb Blood Flow Metab. 2010;30:46-50.

30. Lassen NA, Bartenstein PA, Lammertsma AA, et al. Benzodiazepine receptor quantification in vivo in humans using $\left[{ }^{11} \mathrm{C}\right]$ flumazenil and PET: application of the steady-state principle. J Cereb Blood Flow Metab. 1995;15:152-165.

31. Akaike H. A new look at the statistical model identification. IEEE Trans Automat Contr. 1974; 19:716-723.

32. Carson R. Parameter estimation in positron emission tomography. In: Phelps ME, Mazziotta J, Schelbert HR eds. Positron Emission Tomography and Autoradiography: Principles and Applications for the Brain and Heart. New York, NY: Raven Press; 1986:347-390.

33. Bevington PR, Robinson KD, eds. Data Reduction and Error Analysis for the Physical Sciences. Boston, MA: McGraw-Hill; 2003.

34. Fujimura Y, Zoghbi SS, Simeon FG, et al. Quantification of translocator protein $(18 \mathrm{kDa})$ in the human brain with PET and a novel radioligand, ${ }^{18} \mathrm{~F}$-PBR06. J Nucl Med. 2009;50:1047-1053.

35. Turkheimer FE, Edison $P$, Pavese N, et al. Reference and target region modeling of $\left[{ }^{11} \mathrm{C}\right]-(\mathrm{R})-\mathrm{PK} 11195$ brain studies. $J$ Nucl Med. 2007;48:158-167. 